\title{
Intracranial Subdural Hematoma Following Epidural Analgesia in Obstetrics
}

\author{
Martínez S. ${ }^{1}$, Comps 0. ${ }^{1}$, Medrano S. ${ }^{2}$, Montes A. ${ }^{1}$ \\ ${ }^{1}$ Parc de Salut MAR, Dept of Anaesthesiology \& Intensive Care, Barcelona, Spain, 2Parc de Salut MAR, Neuroradiology, \\ Department of Imaging and Radiodiagnostics, Barcelona, Spain
}

\section{Background}

Post-dural puncture headache (PDPH) is a common complication after neuraxial anesthesia and is frequent in obstetrics. The persistence of headache despite adequate treatment, however,should alert us to the possibility of other more serious causes.

\section{Case}

A 27 years old primigravida with gestational diabetes wellcontrolled. During the first phase of labor, epidural analgesia was administered, with a single puncture at the L4-L5 level. A test dose of $3 \mathrm{ml}$ of $0.25 \%$ bupivacaine plus adrenalin was negative.

The delivery was normal, but 12 hours later the patient reported an isolated fronto-occipital headache of moderate intensity, which increased upon sitting up, with no other symptoms.

She was diagnosed with PDPH and treated according to our hospital's protocol: intravenous paracetamol $(1 \mathrm{~g} / 6 \mathrm{~h})$, metamizole ( $2 \mathrm{~g} / 6 \mathrm{~h})$, hydrocortisone (100 mg/8 h; 3 doses) and oral caffeine (300 mg/8 h).

The headache disappeared until 24 hours later, when the patient complained of a disabling orthostatic headache, with no accompanying symptoms. An epidural blood patch (EBP) was performed with the injection of $20 \mathrm{ml}$ of autologous blood at the L3-L4 level, and the symptoms disappeared immediately.

Twenty-four hours later the patient presented the same persistent headache. A second EBP was performed, but suspended after the administration of $11 \mathrm{ml}$ due to intense lumbar pain during. The patient reported no improvement.

We requested a cranial magnetic resonance (MR), which showed a subdural hematoma (SDH) in the right hemisphere with alterations of cerebrospinal fluid hypotension (Figure 1).

The patient remained hospitalized for a week, with conservative treatment: oral paracetamol, ibuprofen and intravenous tramadol on demand, bed rest, hydration, and consumption of caffeinated beverages. She did not require any change in treatment; symptoms improved steadily and she was discharged 10 days after the birth.

Two months later, the patient was asymptomatic and another MR showed the complete disappearance of the hematoma (Figure 2).
Figure 1
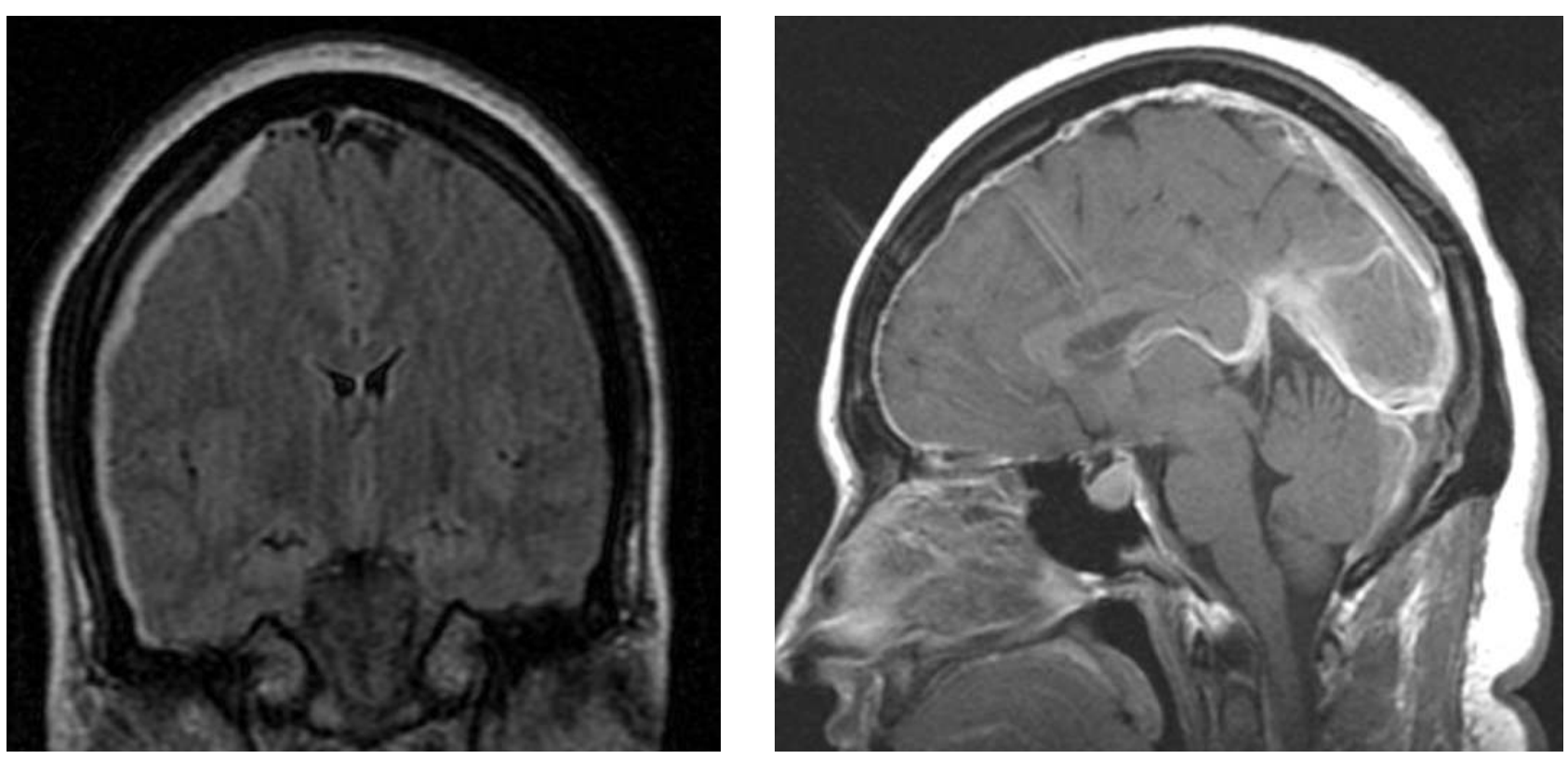

Figure 2
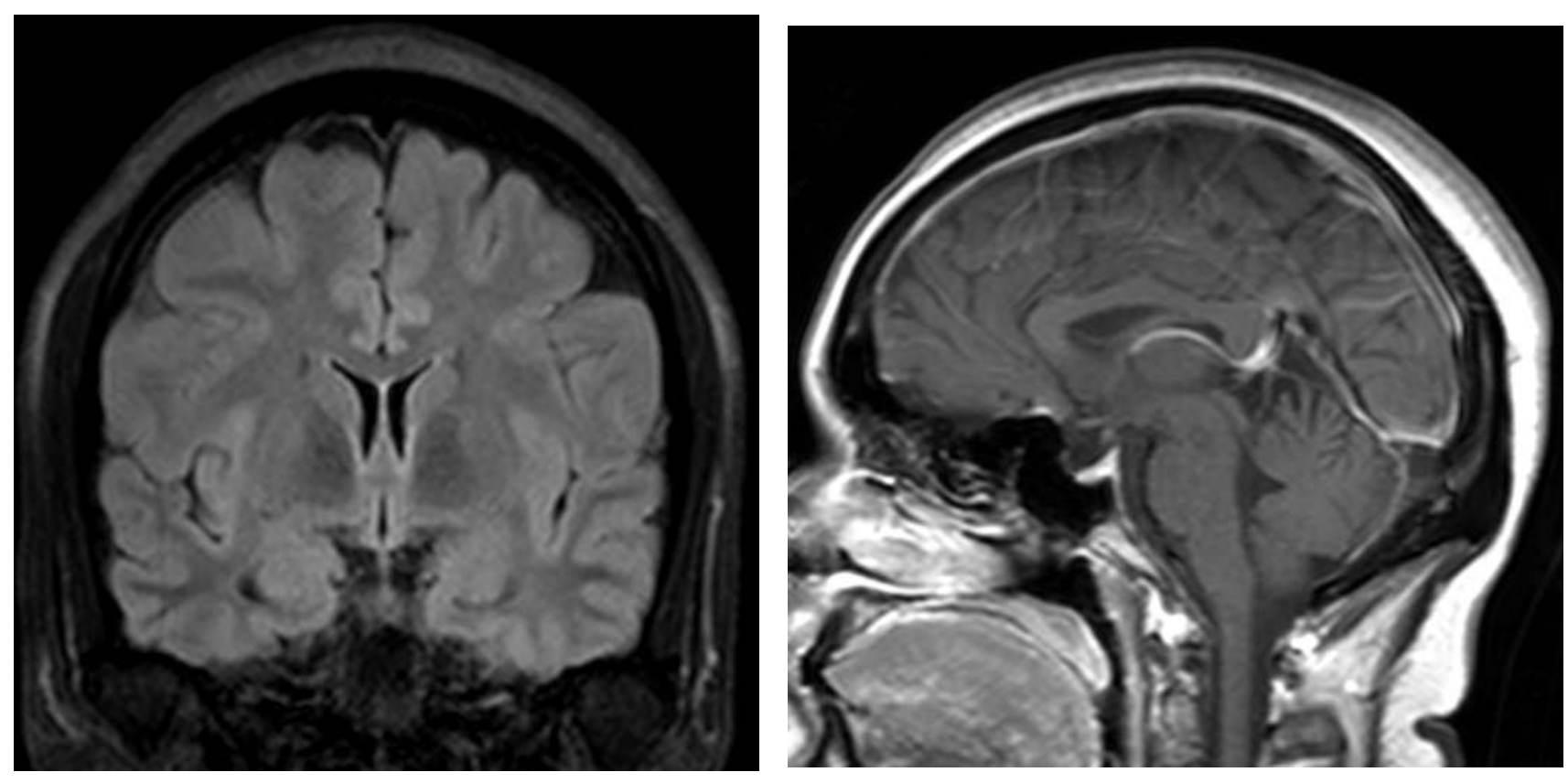

\section{Discussion}

Epidural is used routinely and serious complications are infrequent. After a dural puncture, there is a risk of intracranial SDH.

PDPH may be the only symptom and its persistence despite multiple treatments requires that other causes be ruled out with imaging studies (MR).

It is important to be aware of the risk factors for PDPH and to make a differential diagnosis so that a SDH may be diagnosed and treated quickly.

Early suspicion can prevent neurological consequences.

\section{References}

Turnbull DK,et.al. Br J Anaesth 2003;91:718-729 Bleeker CP,et.al. Br J Anaesth 2004;93:461-464
Amorim JA, et.al. Rev Bras Anestesiol 2010; 60: 620-629.

Zeidan A, et.al. Int J Obstet Anesth 2006; 15: 50-58 